\title{
Abbreviations Used for Frequently Cited Works
}

Agricola, De Inventione: Rudolph Agricola, De Inventione dialectica libri tres, foreword Wilhelm Risse (Cologne, I 528; rpt. Hildesheim: Georg Olms, I 976). Agrippa, De Incertitudine: Henry Cornelius Agrippa, De Incertitudine et vanitate scientiarum et artium, in Opera, vol. 2 (Lyon, I60o [?]; rpt. Hildesheim: Georg Olms, i970).

Alsted, Rhetorica: Johann Heinrich Alsted, Rhetorica (Herborn, 16 I6).

Amyot, Projet: Jacques Amyot, Projet de l'Eloquence royale, composé pour Henry III, roi de France (Versailles: Ph.-D. Pierres, I 805).

Aristotle, Rhetoric: Aristotle, The "Art" of Rhetoric, trans. John H. Freese (Cambridge, Mass.: Harvard University Press, I982).

Bacon, Selected Writings: Francis Bacon, Selected Writings, ed. Hugh G. Dick (New York: Random House, I955).

Barbaro, Della Eloquenza: Daniel Barbaro, Della Eloquenza, in Trattati di poetica e retorica del Cinquecento, ed. Bernard Weinberg (Bari: Laterza. 1970), 2 : 335-45 I.

Bary, Rhetorique: René Bary, La Rhetorique françoise (Paris, I659). Microfilm.

Bulwer, Chirologia: John Bulwer, Chirologia: or the Natural Language of the Hand, in Chirologia: or the Natural Language of the Hand and Chironomia: or the Art of Manual Rhetoric, ed. James W. Cleary (Carbondale: Southern Illinois University Press, I974).

Bulwer, Chironomia: John Bulwer, Chironomia: or the Art of Manual Rhetoric, in Chirologia: or the Natural Language of the Hand and Chironomia: or the Art of Manual Rhetoric, ed. James W. Cleary (Carbondale: Southern Illinois University Press, 1974).

Caussin, Eloquentia sacra: Nicholas Caussin, De Eloquentia sacra et humana, 3d ed. (Paris, I630). Microfilm. 
Cavalcanti, Retorica: Bartolomeo Cavalcanti, La Retorica (Ferrara: Gabriel Giolito, I 559). Microfilm.

Cicero, De inventione: Cicero, De inventione, De optimo genere oratorum, Topica, trans. H. M. Hubbell (London: William Heinemann, I949).

Cicero, De oratore: Cicero, De oratore, trans. E. W. Sutton and H. Rackham (London: William Heinemann, I959).

de' Conti, Dialogus: Anto Maria de' Conti, De eloquentia dialogus, in Trattati di poetica e retorica del Cinquecento, ed. Bernard Weinberg (Bari: Laterza, I 970), 2:I 4I-6I.

Denores, "Breve Trattato dell'Oratore": Giason Denores, "Breve Trattato dell'Oratore," in Trattati di poetica e retorica del Cinquecento, ed. Bernard Weinberg (Bari: Laterza, I970), 3:IOI-34.

Du Pré, Pourtraict: J. Du Pré de la Porte, Le Pourtraict de l'Eloquence Françoise (Paris, I62I).

Du Vair, Traitté: Guillaume Du Vair, Traitté de l'Eloquence Françoise, in Oeuvres (Paris: Sebastien Cramoisy, I64I ; rpt. Geneva: Slatkine Reprints, I 970), 389-410.

Erasmus, Ciceronianus: Desiderius Erasmus, Ciceronianus, in Erasmus von Rotterdam, Ausgewählte Schriften, ed. Werner Welzig, vol. 7 (Darmstadt: Wissenschaftliche Buchgesellschaft, I972).

Fabri, Le grand et vrai art: Pierre Fabri, Le grand et vrai art de pleine rhétorique, ed. A. Héron (Rouen: A. Lestringant, I 890).

Fumaroli, L'âge de l'éloquence: Marc Fumaroli, L'âge de l'éoquence: Rhétorique et "res literaria" de la Renaissance au seuil de l'époque classique (Geneva: Droz, I 980).

Furetière, Nouvelle allégorique: Antoine Furetière, Nouvelle allégorique, ou Histoire des derniers troubles arrivés au royaume d'Eloquence, ed. Eva van Ginneken (Geneva: Droz, I967).

Fuscano, "Della oratoria e poetica facoltà": Giovanni Berardino Fuscano, "Della oratoria e poetica facoltà," Trattati di poetica e retorica del Cinquecento, ed. Bernard Weinberg (Bari: Laterza, I 970), I: $187-95$.

George of Trebizond, Oratio: George of Trebizond (Trapezuntius), Oratio de laudibus eloquentie, in John Monfasani, George of Trebizond: A Biography and a Study of His Rhetoric and Logic (Leiden: E. J. Brill, I976), 365-69.

George of Trebizond, Rhetoricorum: George of Trebizond, Rhetoricorum libri $V$ (Venice: Aldine Press, I 523).

Granada, Ecclesiastica Rhetorica: Luis de Granada, Ecclesiastica Rhetorica (Cologne, I 582). Microfilm.

Guzman, Primera Parte: Joan de Guzman, Primera Parte de la Rhetorica (Alcala de Henares, I 589 l.

Jewel, Oratio: John Jewel, Oratio Contra Rhetoricam, in The Works, ed. John Ayre (Cambridge: Cambridge University Press, I 850), 4:I 283-9I.

Keckermann, Systema: Bartholomew Keckermann, Systema Rhetorices (Hanover, I608).

Le Grand, Discours: M. Le Grand, Discours, in René Bary, La Rhetorique françoise (Paris, I659). Microfilm. 
Melanchthon, Elementorum: Philip Melanchthon, Elementorum Rhetorices Libri Duo (Wittenberg, I5 19). Microfilm.

Melanchthon, Encomion: Philip Melanchthon, Encomion eloquentiae, in Werke in Auswahl, ed. Robert Stupperich /Gütersloh: Gütersloh Verlagshaus Gerd Mohn, I96I), 3:43-62.

Montaigne, Oeuvres: Michel de Montaigne, Oeuvres complètes, ed. Albert Thibaudet and Maurice Rat (Paris: Gallimard, r962).

Müllner: Karl Müllner, ed., Reden und Briefe italienischer Humanisten (Vienna, I 899; rpt. Munich: Wilhelm Fink, I970).

Murphy, Renaissance Eloquence: James J. Murphy, ed., Renaissance Eloquence: Studies in the Theory and Practice of Renaissance Rhetoric (Berkeley: University of California Press, 1983).

Parker, Literary Fat Ladies: Patricia Parker, Literary Fat Ladies: Rhetoric, Gender, Property (London: Methuen, I987).

Patrizi, Della retorica: Francesco Patrizi, Della retorica dieci dialoghi (Venice, I 562). Microfilm.

Peacham, Garden: Henry Peacham, The Garden of Eloquence (1593), intro. William G. Crane (Gainesville, Fla.: Scholars' Facsimiles \& Reprints, I954).

Pico, Epistola: Giovanni Pico della Mirandola, Epistola Hermolao Barbaro, in Giovanni Pico della Mirandola and Gian Francesco Pico, Opera Omnia, intro. Cesare Vasoli (Basel, I 557; rpt. Hildesheim: Georg Olms, I 969), 35 I-58.

Plett, Rhetorik der Affekte: Heinrich F. Plett, Rhetorik der Affekte: Englische Wirkungsästhetik im Zeitalter der Renaissance (Tübingen: Max Niemeyer, 1975).

Prosatori latini: Prosatori latini del Quattrocento, ed. Eugenio Garin (Milan: Riccardo Ricciardi, I952).

Puttenham, Arte: George Puttenham, The Arte of English Poesie, intro. Baxter Hathaway (Kent, Ohio: Kent State University Press, I970).

Quintilian, Institutio: Quintilian, Institutio oratoria, trans. H. E. Butler (London: William Heinemann, I963).

Rainolde, Foundacion: Richard Rainolde, The Foundacion of Rhetorike (London, I563; rpt. Amsterdam: Da Capo, I969).

Rainolds, Oratio: John Rainolds, Oratio in laudem artis poeticae, ed. William Ringler and Walter Allen, Jr. (Princeton: Princeton University Press, I 940).

Rainolds, Oxford Lectures: John Rainolds, Oxford Lectures on Aristotle's "Rhetoric," ed. Lawrence D. Green (Newark: University of Delaware Press, I986).

Ramus, Attack on Cicero: Peter Ramus, Attack on Cicero: Text and Translation of Ramus's "Brutinae Quaestiones," ed. James J. Murphy, trans. Carole Newlands (Davis, Calif.: Hermagoras Press, I992).

Ramus, Dialectique: Pierre de la Ramée (Ramus), Dialectique, in Gramere (I562), Grammaire (I 572), Dialectique (I555) (Paris; rpt. Geneva: Slatkine Reprints, I972).

Regius, Panegyricus: Raphael Regius, De Laudibus eloquentiae panegyricus (Venice, I485).

Shuger, Sacred Rhetoric: Debora K. Shuger, Sacred Rhetoric: The Christian 
Grand Style in the English Renaissance (Princeton: Princeton University Press, I988).

Sidney, Apology: Sir Philip Sidney, An Apology for Poetry, ed. Forrest G. Robinson (Indianapolis: Bobbs-Merrill, 1970).

Soarez, De Arte: Cypriano Soarez, De Arte Rhetorica Libri Tres (Verona, I589). Microfilm.

Speroni, Dialogo: Sperone Speroni, Dialogo della rettorica, in Dialogo della lingua e Dialogo della rettorica, intro. Giuseppe De Robertis (Lanciano: R. Carabba, I9I 2), 85-I40.

Vives, De Causis: Juan Luis Vives, De Causis Corruptarum Artium, in Opera Omnia, vol. 6, ed. Francisco Fabian y Fuero (Valencia, 1745; rpt. London: Gregg Press, I964).

Vives, De Ratione: Juan Luis Vives, De Ratione dicendi, in Opera Omnia, vol. 2. Wilson, Arte: Thomas Wilson, The Arte of Rhetorique (1560), ed. G. H. Mair (Oxford: Clarendon Press, 1909). 